\title{
Data storage and DNA banking for biomedical research: technical, social and ethical issues
}

\author{
Recommendations of the European Society of Human Genetics
}

European Journal of Human Genetics (2003) 11, Suppl 2, S8-S10. doi:10.1038/sj.ejhg.5201115

\section{Introductory considerations}

The last few years have witnessed an important expansion of human DNA sampling and data collecting. This activity has strategic importance for genetic research, clinical care and future treatments. Collections should be maintained and their use optimized. Human DNA, tissue or cell collections and the attached databases have been extensively exchanged for scientific purposes. However, the status of such collections and databases is not well defined and most institutions have no written policies or agreements regarding this activity.

Regulations are at an early stage in most European countries and, with the multiplicity of actors and of rules that regulate them, the situation is difficult to comprehend. The rules for exchanging and sharing of information and material are not clear. The notion of return of benefits to research subjects or communities is fairly recent.

Collections and databases are expensive to establish and maintain. They are of high interest to scientists, to industrial partners, to health care professionals and ultimately for the community. It is difficult to separate public and private research, as researchers from both sectors are often involved in the same project. While this enables effective technology transfer, it also gives rise to concerns about conflicts of interest. There is a need to promote confidence in research based on DNA collections.

To discuss these issues and produce recommendations from the professional point of view, the Public and Professional Policy Committee (PPPC) of the European Society of Human Genetics (ESHG) organized a workshop in April 2000 in Paris, France, to which 51 experts from 15 European countries were invited. Prior to the meeting, they received a working paper developed by the PPPC, which was revised after the meeting to take into account the points of views expressed by the participants.

Following the workshop, the ESHG issued statements and recommendations, which were endorsed by the membership. They are expected to reflect the views of the scientific community.

\section{Statements and recommendations Reasons for DNA banking}

(1) Healthcare depends on research, and modern research requires access to biological samples, including DNA. The potential benefits justify the establishment of DNA banks, but the possibility of misuses imposes a responsibility of proper management and protection of the subjects' interests.

\section{Status of collections}

(2) There are several types of status for collections. In anonymous collections, the biological materials were originally collected without identifiers and are impossible to link with their sources. In anonymized collections, biological materials were originally identified, but have been irreversibly stripped of all identifiers and are impossible to link to their sources. In identifiable collections, biological materials are unidentified for research purposes, but can be linked to their sources through the use of a code. In identified collections, identifiers, such as a name, patient number or clear pedigree location, are attached to the biological materials.

\section{Consent requirements for new collections}

(3) Informed consent is required for all types of DNA banking. Where it is for research use, ethical committee oversight is required to ensure an acceptable balance between risks and benefits.

(4) Consent should be written and specific protections should be provided for vulnerable subjects and vulnerable populations, based on the general principle of acting in their best interest.

(5) Individuals should be informed with respect to the types of research that will or might be carried out, the arrangements for access to or sharing of stored samples, and the duration of storage.

(6) Consent should be freely given, free from pressure, based on the information provided by trained staff. 
(7) Individuals should be given the right to withdraw at any time from the research, including destruction of their sample.

(8) As it is difficult to foresee all the potential research applications that a collection may be used for, individuals may be asked to consent for a broader use. In that case, there is no need to recontact individuals although the subjects should be able to communicate should they wish to withdraw.

\section{Consent requirements for existing collections}

(9) Regarding already stored anonymous samples, irreversibly unlinked to the name, these may be used for other purposes than those originally intended.

(10) The decision to strip samples of identifiers irreversibly needs careful consideration. The benefit of having unlinked anonymized samples is to secure absolute confidentiality and thereby allows further use of the samples. However, retaining identifiers, while requiring further consent from the subject, will permit more effective biomedical research and the possibility of recontacting the subject when a therapeutic option becomes available.

(11) Anonymized samples are acceptable in order to allow sample and information sharing for research purpose with minimum risk. Anonymization techniques should be standardized to ensure their robustness. Demographic and clinical data attached to anonymized samples should be coded with international nomenclatures wherever possible.

(12) In the case of existing collections, investigators should be required to recontact subjects to obtain consent for new studies. If it is impracticable to gain consent, an appropriate ethics review board should give its consent for further use of the samples based on the notion of minimum risk for the donor.

(13) Concerning postmortem uses of samples, a policy of unrestricted access cannot be justified on the grounds that the risk or harm for the subject are no more an issue. If individuals restrict use of their sample when they are still alive, those restrictions apply after their death.

(14) Old collections should be regarded as abandoned and therefore useable for new research purposes as long as ethics committee approval is obtained.

\section{Consent requirements in population studies}

(15) If a population is to be the subject of research, additional consent may be required at a group level through its cultural appropriate authorities. The precise form of the consent must take cultural differences into account and respect minority rights.

(16) If the sampling is done by a group from a different country, regulations from both the country of origin of the samples and the country of origin of the researchers should be respected in order to maximize the protection of the rights of the sampled population.
Management, quality control and security issues

(17) The value of a collection is proportional to the amount and quality of the information attached to it. The full benefits for which the subjects gave their samples will be realized through maximizing collaborative high-quality research. Therefore, there is an ethical imperative to promote access and exchange of information, provided confidentiality is protected. This includes availability of catalogues.

(18) The implementation of security mechanisms to ensure the confidentiality and long-term conservation of genetic information are an absolute condition. These mechanisms should be in place before the sampling is done, including standardization of coding, sample tracking, computerization and encryption. The standards adopted should permit sample information sharing for research purpose with minimum risk. Discussions should be encouraged among consortia of bankers to issue standardized bank protocols.

(19) Banks, collections and databases should receive some form of authorization from institutions. Means of ensuring oversight may vary.

(20) Authorization cannot occur without appropriate funding in place to ensure security, data protection and the ability to remove samples if requested.

(21) The custodians of the banks have to balance conservation of the banked samples against the distribution to research collaborators based on a policy statement.

(22) Professionals involved in banking and storing genetic data should have a written protocol describing the rights and obligations of all parties with respect to storage.

(23) Provision should be specified to ensure continued care of the collection under any circumstances.

(24) In the case of academically based banks, governmental agencies should advise local institutional review board to follow specified guidelines to ensure that DNA banks adhere to uniform standards.

(25) Commercial DNA banks should adhere to a core set of rules.

\section{Access/sharing/ownership}

(26) While protecting confidentiality, the free circulation and the availability of genetic information and samples for research should be promoted.

(27) Agreements about ownership of samples and data, and access to biological material and information should be determined by multiparty contracts and not regulated by legislation. Practices should be based on the following principles:

(a) With respect of anonymized and identifiable data, the subject should always be considered as a primary controller of its DNA and clinical information directly derived from it. Once the information has been 
processed, it becomes research data (ie data) unless there is agreed private ownership. The processor and/or principle investigator of DNA sample and genetic data should be considered as the custodian of the DNA/ genetic data. As such, he has to take all the appropriate steps to protect the data, its storage, use and access. It follows that intellectual property would be of the researcher but with due consideration for benefit sharing.

(b) With respect of anonymous data, the DNA samples/ genetic data should be considered as abandoned data. As such, the processor and/or Principal investigator should be considered as the custodian of these data and should take any steps to protect the data, its storage, use and access. It follows that the intellectual property would be of the researcher but with due consideration for benefit sharing.

(c) While a property position may allow for actual or potential financial return, the gift relationship approach to research avoids individual returns but not the possibility of commercialization by the researcher, through traditional intellectual property rules.

(28) Use of collections by third parties should be allowed, provided there is no transfer of ownership and the use is in agreement with the present guidelines.
This document forms part of a BIOTECH programme financed by the Commission of the European Communities (CEE BIO4-CT98-0550).

All rights reserved. No part of this document may be reproduced or utilized in any forma or by any means, electronic or mechanical, including photocopying, recording, microcoping or any information storage and retrieval system, without in writing from the copyright holder.

\section{(C) Copyright 2001 by ESHG/PPPC}

*Members of the PPPC were Ségolène Aymé (Paris, France), Martin Bobrow (Cambridge, UK), Jean-Jacques Cassiman (Leuven, Belgium), Gerry Evers-Kiebooms (Leuven, Belgium), Peter Farndon (Birmingham, UK), Helena Kääriäinen (Helsinki, Finland), Ulf Kristoffersson (Lund, Sweden), Marcus Pembrey (London, UK), Sandy Raeburn (Nottingham, UK), Jörg Schmidtke (Hannover, Germany), Leo ten Kate (Amsterdam, Netherlands) and Lisbeth Tranebjaerg (Copenhagen, Denmark).

**The workshop was organized by Ségolène Aymé (Paris, France), Jean-Jacques Cassiman (Leuven, Belgium) and Jörg Schmidtke (Hannover, Germany). 